\title{
Corrigendum to A High-Throughput Approach to Identify Specific Neurotoxicants / Developmental Toxicants in Human Neuronal Cell Function Assays
}

\author{
Johannes Delp1,2,3, Simon Gutbier1,2, Stefanie Klima1,2,3, Lisa Hoelting1, Kevin Pinto-Gil4, Jui-Hua Hsieh5, \\ Michael Aichem 6 , Karsten Klein 6 , Falk Schreiber 6,7, Raymond R. Tice ${ }^{8}$, Manuel Pastor ${ }^{4}$, Mamta Behl 8 and \\ Marcel Leist ${ }^{1}$ \\ ${ }^{1}$ In vitro Toxicology and Biomedicine, Dept inaugurated by the Doerenkamp-Zbinden Foundation, University of Konstanz, Konstanz, Germany; ${ }^{2}$ Research \\ Training Group RTG1331, University of Konstanz, Konstanz, Germany; ${ }^{3}$ Cooperative doctorate college InViTe, University of Konstanz, Konstanz, \\ Germany; ${ }^{4}$ Research Program on Biomedical Informatics (GRIB), Dept. of Experimental and Health Sciences, Universitat Pompeu Fabra, Barcelona, \\ Spain; ${ }^{5}$ Kelly Government Solutions, Durham, NC, USA; ${ }^{6}$ Department of Computer and Information Science, University of Konstanz, Konstanz, \\ Germany; ${ }^{7}$ Faculty of Information Technology, Monash University, Melbourne, Australia; ${ }^{8}$ Division of National Toxicology Program, National Institute of
} Environmental Health Sciences, Research Triangle Park, NC, USA

In this manuscript, which appeared in ALTEX 35, 235-253 (doi:10.14573/altex.1712182), the Acknowledgements should read:

\section{Acknowledgements}

This work was supported by the Land BW, the Doerenkamp-Zbinden Foundation, the DFG (RTG1331, KoRS-CB), the BMBF (NeuriTox), and it has received funding from the European Union's Horizon 2020 research and innovation programme under grant agreement No. 681002 (EU-ToxRisk).

doi:10.14573/altex.1904111

\section{Corrigendum to Normalization of Data for Viability and Relative Cell Function Curves}

Alice Krebs 1,2, Johanna Nyffeler 1,3, Jörg Rahnenführer 4 and Marcel Leist 1,2,5

${ }^{1}$ In vitro Toxicology and Biomedicine, Dept inaugurated by the Doerenkamp-Zbinden Foundation, University of Konstanz, Konstanz, Germany; ${ }^{2}$ Konstanz Research School Chemical Biology (KoRS-CB), University of Konstanz, Konstanz, Germany; ${ }^{3}$ present address: National Centre for Computational Toxicology, US EPA, Research Triangle Park, NC, USA; ${ }^{4}$ Department of Statistics, TU Dortmund University, Dortmund, Germany; ${ }^{5}$ CAAT-Europe, University of Konstanz, Konstanz, Germany

In this manuscript, which appeared in ALTEX 35, 268-271 (doi: 10.14573/1803231), the Acknowledgements should read:

\section{Acknowledgements}

This work was supported by BMBF and DFG (KoRS-CB) grants, and it has received funding from the European Union's Horizon 2020 research and innovation programme under grant agreement No. 681002 (EU-ToxRisk).

doi:10.14573/altex.1904113 